\title{
Surgical, Orthodontic and Prosthodontic Rehabilitation of a Patient with Follicular Ameloblastoma: A Case Report
}

\author{
Herman F. Sailer ${ }^{a}$ \\ Fadi Tarawneh ${ }^{b}$ \\ Panagiotis Fourkas ${ }^{b}$ \\ Dimitrios Z. Antoniades ${ }^{c}$ \\ Athanasios E. Athanasiou ${ }^{d}$
}

\section{ABSTRACT}

This case report describes the combined surgical, orthodontic and prosthodontic rehabilitation of an adult female patient with a previous history of follicular ameloblastoma, which was treated through partial mandibulectomy and an immediate replacement of missing bone with an autologous calvarial bone graft. Orthodontic treatment was undertaken in order to restore occlusal disturbances and obtain sufficient space for two dental implants and an optimum prosthodontic rehabilitation. (Eur J Dent 2010;4:192-196)

Key words: Follicular ameloblastoma; Bone graft; Mandibulectomy; Orthodontic treatment.

a Professor of Oral and Maxillofacial Surgery, Klinik Professor Sailer, Zurich, Switzerland.

b Orthodontic specialist, Former resident, Department of Orthodontics, Aristotle University of Thessaloniki, Thessaloniki, Greece.

c Professor and Chairman, Department of Oral Medicine/ Pathology, School of Dentistry, Aristotle University of Thessaloniki, Thessaloniki, Greece.

d Professor and Chairman, Department of Orthodontics, School of Dentistry, Aristotle University of Thessaloniki, Thessaloniki, Greece.

- Corresponding author: Athanasios E. Athanasiou Department of Orthodontics, School of Dentistry, Aristotle University of Thessaloniki, GR-54124

Thessaloniki, Greece.

Phone: +302310999556

Fax: +30 2310999549

E-mail: aeathandadent.auth.gr

\section{INTRODUCTION}

Ameloblastoma is a benign but locally aggressive neoplasm of the odontogenic epithelium. ${ }^{1}$ Accounting for $11 \%$ of all odontogenic tumors, ameloblastoma is the most common odontogenic neoplasm affecting the jaws, yet it only accounts for $1 \%$ of all tumors of the maxilla and mandible. ${ }^{2-4}$ The average age of patients presenting with ameloblastoma is 36 years, with men and women being equally affected. ${ }^{5}$ Ameloblastomas are classified as solid/multicystic, intraosseous, or unicystic, with peripheral subtypes. ${ }^{6,7}$ The radiographic appearance of ameloblastoma, dentigerous cysts, and odontogenic keratocysts is similar and for this reason a biopsy is recommended to obtain a pre- 
cise diagnosis of ameloblastoma. ${ }^{8}$ Although a benign tumor, ameloblastoma is aggressive because of the myeline nature of its growth and because its conservative treatment is associated with a high rate of recurrence (50\%-90\%). Primary resection of this benign tumor is, therefore, considered the only predictable curative form of therapy.-11 Unfortunately, this treatment leaves the patient with a defect in the affected region of the jaw. A multidisciplinary approach is needed for the patient's complete rehabilitation, including bone grafting, the planned placement of implant, and the prosthetic work. Sometimes the orthodontist's role in this multidisciplinary approach is to create an optimum occlusal relationship and sufficient space to allow for successful reconstruction of the affected region of the jaw.

\section{CASE REPORT}

Diagnosis and etiology

A 31-year-old Caucasian female was referred to the Graduate Orthodontic Clinic of Aristotle University of Thessaloniki following mandibulectomy and immediate replacement of removed bone with autologous calvarial bone graft for the purposes of evaluation and treatment of occlusal disturbances. The patient's medical/dental history referred to a tumor, which was histologically classified as follicular ameloblastoma. According to the history, the tumor was located in the posterior right mandibular region encompassing three teeth, specifically the first and second premolars and the first molar. The first author performed a partial mandibular resection and an immediate reconstruction utilizing bone graft taken from the patient's calvaria. All three teeth were removed along with the tumor (Figure 1).

Extraoral examination revealed an orthognathic profile without facial asymmetry and with the lips competent at rest (Figure 2). Intraoral examination revealed amalgam restorations in all maxillary and mandibular second molars, in the left first maxillary premolar, and the left mandibular first molar. Clinical examination indicated satisfactory dental hygiene and periodontal condition, with absence of bleeding on probing and no significant pocket depths. An analysis of dental casts revealed a Class I malocclusion characterized by a $3 \mathrm{~mm}$ overjet, $3 \mathrm{~mm}$ of overbite, moderate crowding in the anterior region of both dental arches 13 $\mathrm{mm}$ crowding in the maxillary dental arch and 6 $\mathrm{mm}$ in the mandibular one, respectively), a labially positioned mandibular right canine, a $2 \mathrm{~mm}$ deviation of the lower dental arch midline to the right, moderate Bolton tooth size discrepancy (mandibular anterior relatively larger than maxillary ones), and an edentulous space due to the aforementioned surgical procedure (Figure 2).

Panoramic radiograph showed no pathology. Analysis of the cephalometric radiograph indicated harmonious sagittal and vertical skeletal relationships (SNA: $83^{\circ}$, SNB: $80^{\circ}$, facial angle: $90^{\circ}$, ANB: $3^{\circ}$, individual ANB: $3.5^{\circ}$ ) (Figure 3 ).

\section{Treatment objectives and alternatives}

The problem list for this patient included the presence of a Class I malocclusion characterized by a condition of moderate crowding in the anterior region of both dental arches, a labially posi-

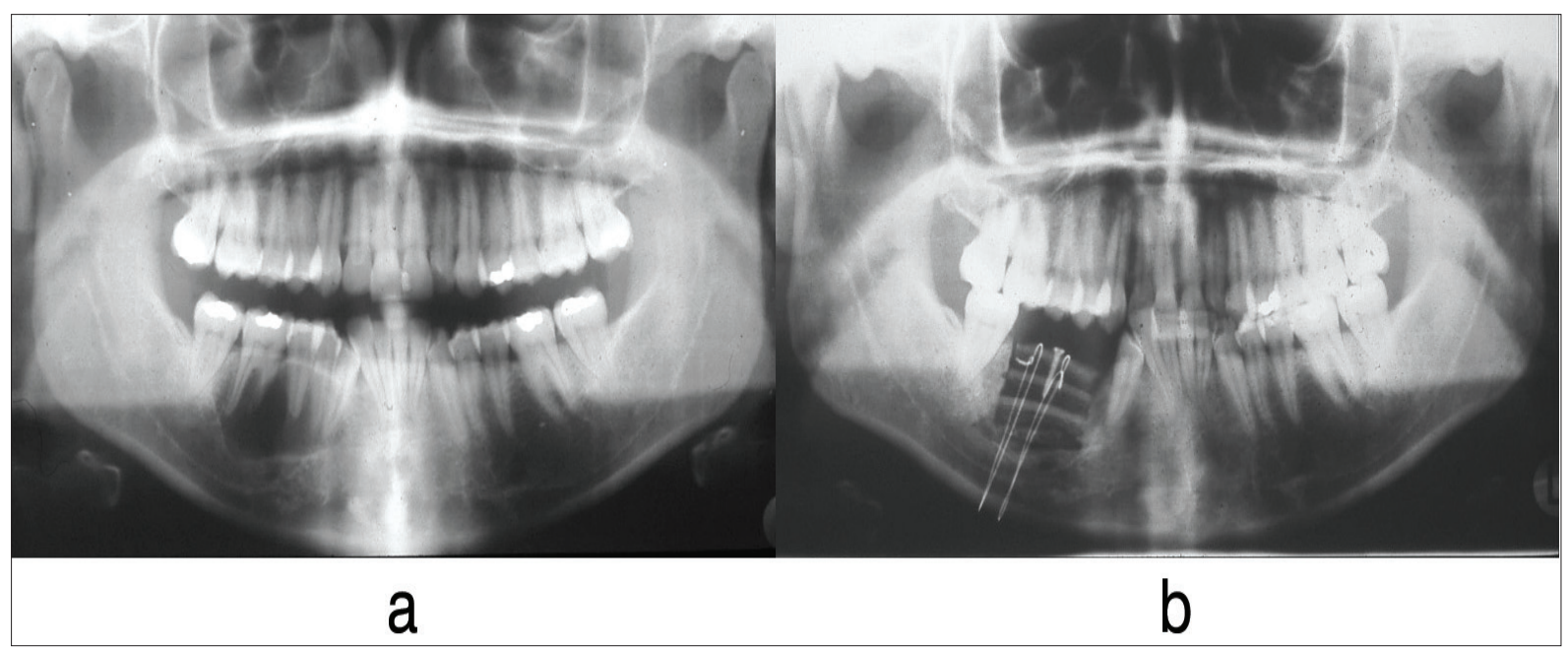

Figure 1. Panoramic radiographs: a. initial radiograph showing the tumor in the right lower region; b. following partial mandibular resection and immediate reconstruction using calvarial bone graft. 
tioned mandibular right canine, a mild deviation of the mandibular dental arch midline to the right, a moderate tooth size discrepancy, and an edentulous space in the right posterior area of the mandible due to the extractions of the right premolars and first molar. The patient's chief complaint referred to the need for optimum prosthetic rehabilitation and a desire for improvement of dental esthetics.

A comprehensive orthodontic treatment aiming to correct the above-mentioned occlusal problems was proposed and accepted by the patient.

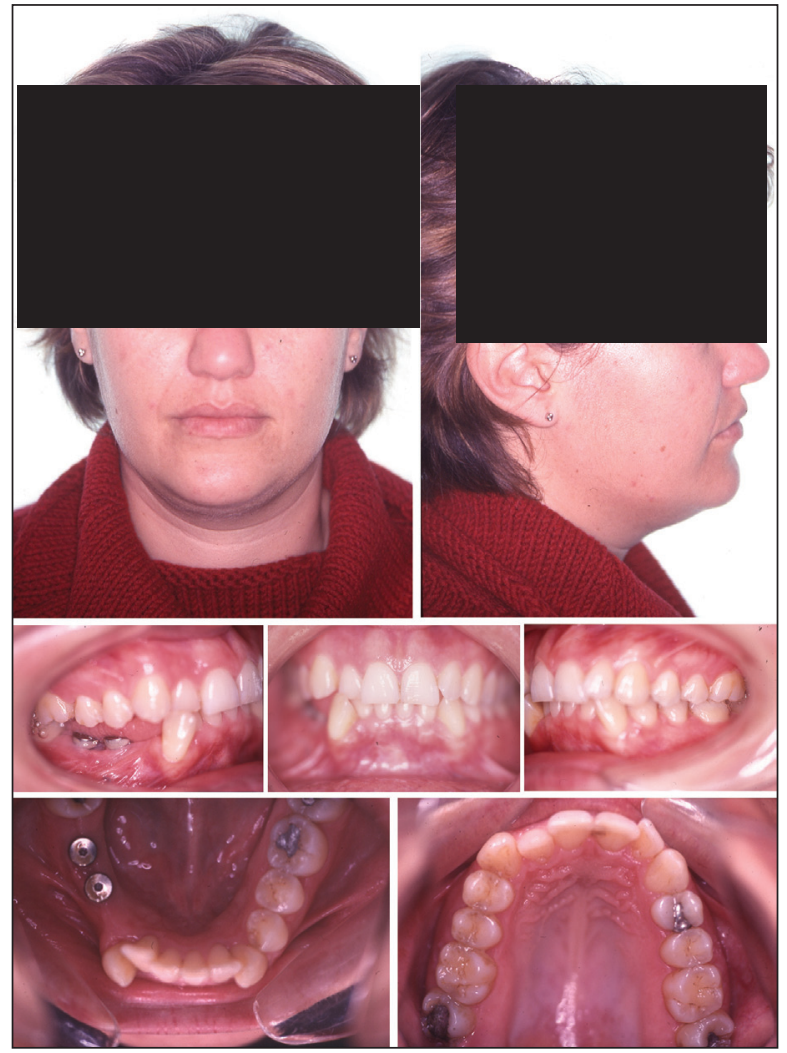

Figure 2. Pre-treatment extraoral and intraoral photographs.

\section{Treatment progress}

The placement of the two dental implants took place by the first author prior to the orthodontic treatment and according to the orthodontic treatment plan. A setup of the patient's dental casts was made to facilitate accurate determination of the exact positions of the two implants. Following their placement, a lingual arch was banded on the lower dental arch for anchorage and space preservation. Fixed appliances (.018" X .022", Roth) were bonded on the maxillary teeth in order to level and align the dental arch. Following the placement of a bracket, the mandibular right canine was uprighted and brought into the dental arch by means of a T-loop. Then, fixed appliances were bonded to the remaining mandibular teeth and reproximation took place on the lower incisors. The fixed appliances were removed after alignment and leveling of both dental arches, crowding alleviation, and correction of dental arch midlines. Essix ${ }^{\circledR}$ maxillary and mandibular splints were used for retention until the placement of prostheses (Figure 4). New Essix $\AA$ mandibular retainer was delivered following the end of prosthetic work. Due to the patient's advanced stage of pregnancy ( $9^{\text {th }}$ month) and the relevant inconvenience, at this stage it was not possible to place a bonded lingual retainer in the lower anterior teeth, as was originally planned.

\section{Treatment results}

The active orthodontic treatment utilizing fixed appliances in both dental arches lasted 11 months. Superimposition of the initial and final tracings of the lateral cephalometric X-rays indicated that slight labial proclination of the upper

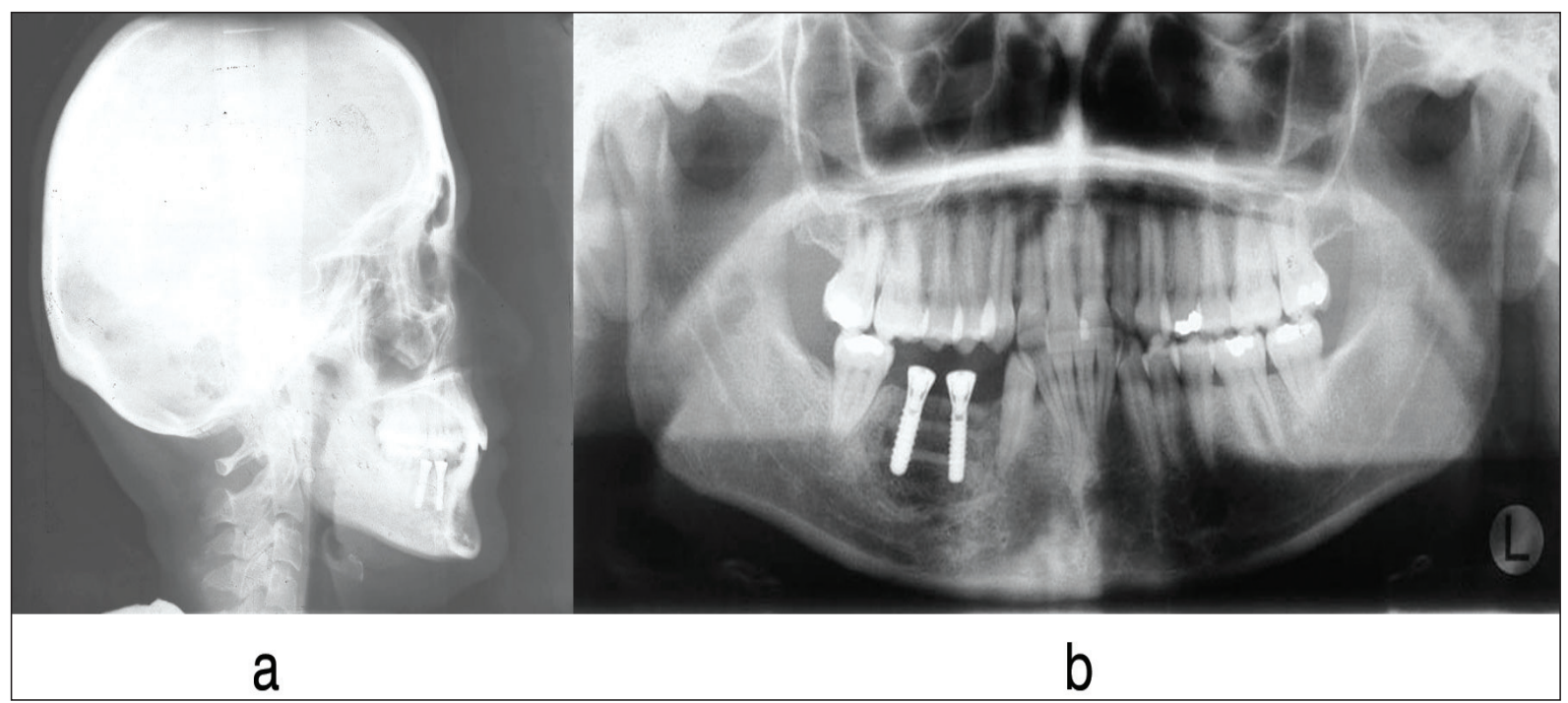

Figure 3. Pre-treatment X-rays, after implant placement: a. cephalometric radiograph; b. panoramic radiograph. 
and lower incisors occurred post-treatment (Figure 5). Prosthodontic rehabilitation of the partially edentulous right mandibular dental arch region was achieved through the placement of two implants and two crowns, respectively (Figure 6).

\section{DISCUSSION}

Ameloblastoma is a benign odontogenic tumor arising from the residual epithelial components of tooth development. It is a slow growing, locally aggressive tumor capable of causing facial deformity, with a high recurrence rate due its capacity to infiltrate trabecular bone. The treatment of ameloblastoma varies from curettage to en block resection. Bone grafts replace the surgically removed bone, with autologous bone grafting being the most desirable. It is typically harvested from intraoral sources (e.g., chin) or extraoral sources (e.g., iliac crest, fibula, calvarial bone).

The most commonly used graft material for alveolar ridge reconstruction is free autogenous iliac bone. ${ }^{12}$ In this case, however, autologous calvarial bone grafts were used to reconstruct the missing

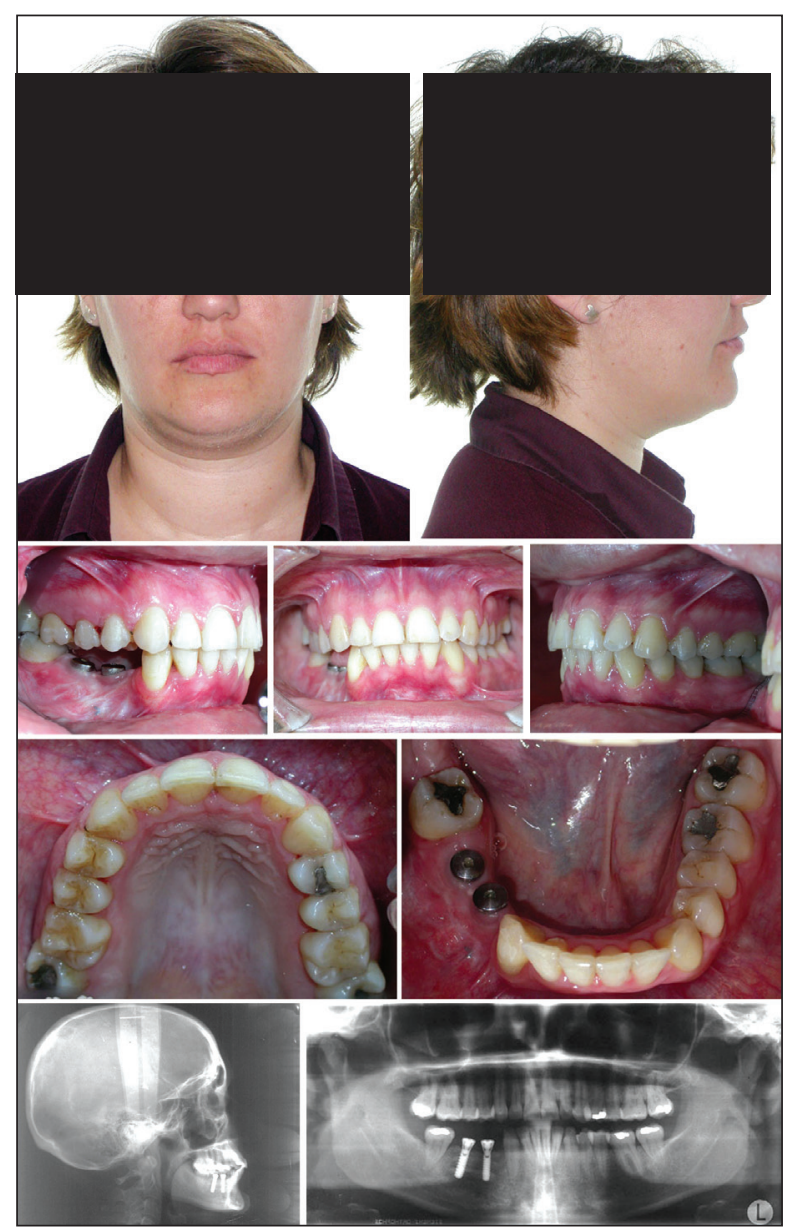

Figure 4. Post-orthodontic treatment photographs and X-rays. mandibular bone following the surgical resection of the tumor and the removal of three teeth in the region. The advantages of calvarial bone grafting include good integration, absence of pain from the donor site, and no visible scar. These advantages, however, are not applicable in the case of thin calvaria bone with a thickness of less than $5 \mathrm{~mm} .{ }^{12}$ Recent reports on the use of calvarial bone grafting for the reconstruction and subsequent placement of dental implants have presented good clinical outcomes, with low rates of graft resorption and high implant survival rates. ${ }^{13-16}$ The results of these studies have showed that calvarial bone grafting appears to be less prone to resorption than iliac grafts are.

In this case, complete functional rehabilitation of the patient included the replacement of the lost three teeth. This goal could have been achieved by the placement of two implants and a bridge, replacing all three teeth. However, this treatment plan would not have addressed the patient's chief complaint, nor would it result in optimum functionality and esthetics. Accordingly, the place-

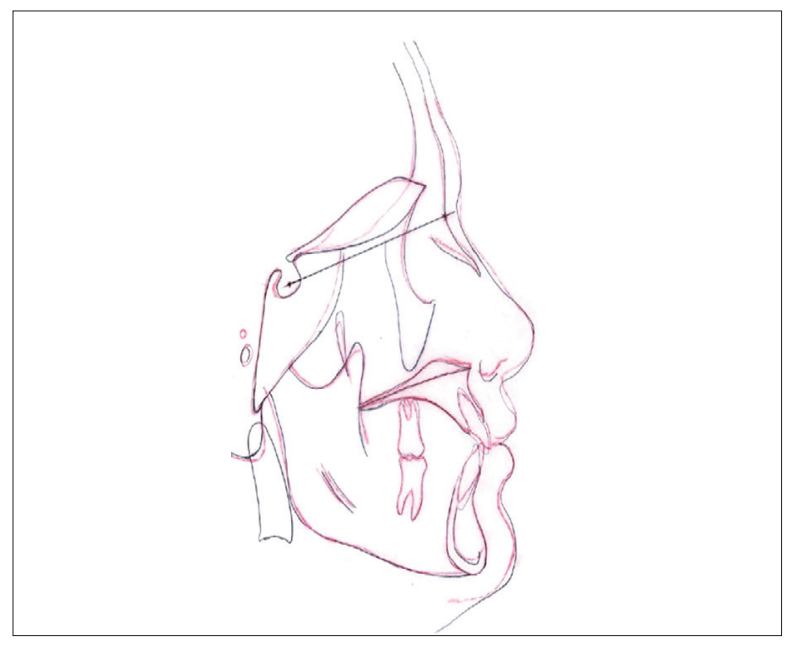

Figure 5. Overall superimposition of initial and final lateral cephalometric tracings.

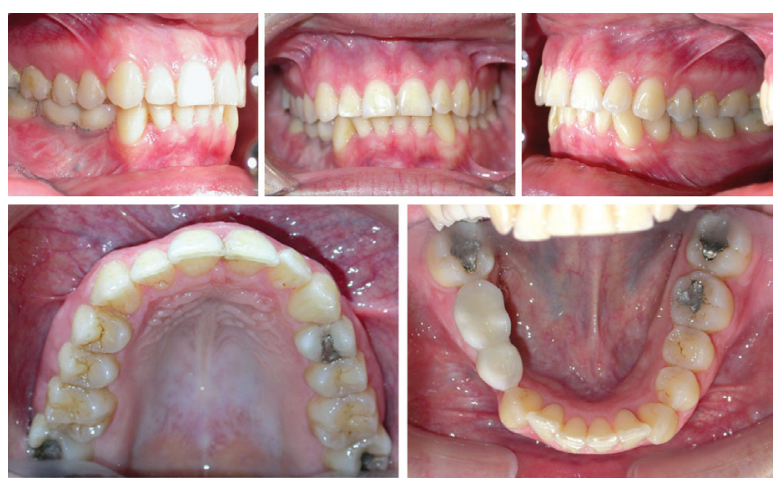

Figure 6. Post-treatment photographs. 
ment of the two implants was decided in relation to the orthodontic treatment plan, aiming for an optimum result. The two implants were placed in the posterior region of the edentulous area, hence replacing only two of the missing teeth, with the extra space being used to correct crowding and to improve dental occlusion.

The outcome of this interdisciplinary approach was the satisfactory restoration of both occlusal function and esthetics.

\section{CONCLUSIONS}

A multidisciplinary approach, including oral surgery, orthodontics, and prosthodontics was able to provide a patient diagnosed with follicular ameloblastoma in the right mandibular region with a satisfactory occlusion following partial mandibular resection and removal of adjacent teeth in the affected site.

\section{REFERENCES}

1. Junquera L, Ascani G, Vicente JC, Garcia-Consuegra L, Roig P. Ameloblastoma revisited. Ann Otol Rhinol Laryngol 2003;112:1034-1039.

2. Gorlin RJ, Chaudhry AP, Pindborg JJ. Odontogenic tumors: Classification, histopathology clinical behaviour in man and domesticated animals. Cancer 1961;14:73-101.

3. Adekeye EO. Ameloblastoma of jaws: A survey of 109 Nigerian patients. J Oral Surg 1980;38:36-41.

4. Adekeye EO, Lavery KM. Recurrent ameloblastoma of the maxillofacial region. Clinical features and treatment. $J$ Maxillofac Surg 1986;14:153-157.

5. Reichart PA, Philipsen HP, Sonner S. Ameloblastoma: biological profile of 3677 cases. Eur J Cancer B Oral Oncol 1995;31:86-99.

6. Ghandhi D, Ayoub AF, Pogrel MA, MacDonald G, Brocklebank LM, Moos KF. Ameloblastoma: a surgeon's dilemma. J Oral Maxillofac Surg 2006;64:1010-1014.

7. Carlson ER, Marx RE. The ameloblastoma: primary, curative surgical management. J Oral Maxillofac Surg 2006;64:484-494.

8. Peterson LJ, Ellis E, Hupp J, Tucker MR. Contemporary oral and maxillofacial surgery. St. Louis: Elsevier Health Sciences 2002 p.345-357.

9. Shatkin S, Hoffmeister FS. Ameloblastoma: a rational approach to therapy. Oral Surg Oral Med Oral Pathol 1965;20:421-435.

10. Sehdev MK, Huvos AG, Strong EW, Gerold FP, Willis GW. Ameloblastoma of maxilla and mandible. Cancer 1974;33:324-333.
11. Müller H, Slootweg PJ. The ameloblastoma, the controversial approach to therapy. J Maxillofac Surg 1985;13:79-84.

12. Gleizal AM, Beziat JL. Maxillary and mandibular reconstruction using bicortical calvarial bone grafts: a retrospective study of 122 reconstructions in 73 patients. Plast Reconstr Surg 2007;119:542-548.

13. lizuka T, Smolka W, Hallermann W, Mericske-Stern R. Extensive augmentation of the alveolar ridge using autogenous calvarial split bone grafts for dental rehabilitation. Clin Oral Implants Res 2004;15:607-615.

14. Bianchi AE, Vinci R, Torti S, Sanfilippo F. Atrophic mandible reconstruction using calvarial bone grafts and implantsupported overdentures: radiographic assessment of autograft healing and adaptation. Int J Periodontics Restorative Dent 2004;24:334-343.

15. Smolka W, Bosshardt DD, Mericske-Stern R, lizuka T. Reconstruction of the severely atrophic mandible using calvarial split bone grafts for implant-supported oral rehabilitation. Oral Surg Oral Med Oral Pathol Oral Radiol Endod 2006;101:35-42.

16. Gratz KW, Sailer HF, Haers PE, Oechslin CK. Mandibular reconstruction with full thickness calvarial bone and temporal muscle flap. Br J Oral Maxillofacial Surg 1996;34:379385. 